\title{
Coronary Artery Spasm Following Off-Pump Coronary Artery Bypass Surgery
}

\author{
Shinya UnaI, ${ }^{1}$ MD, Hitoshi Hirose, ${ }^{1}$ MD, Gary CooK, ${ }^{1}$ PA-C, Yangsin LeE, ${ }^{2}$ MD, Sumio Miura, ${ }^{2}$ MD, \\ Ikutaro KigawA, ${ }^{3} \mathrm{MD}$, Sachito FukUdA, ${ }^{2} \mathrm{MD}$, and Takeshi MiYairi, ${ }^{4} \mathrm{MD}$
}

\begin{abstract}
SUMMARY
Coronary artery spasm after coronary artery bypass surgery may result in life-threatening arrhythmias, circulatory collapse, or death. We report two cases of coronary artery spasm after coronary artery bypass surgery, one of which developed ventricular fibrillation requiring extracorporeal membrane oxygenation support. Both patients were discharged in good condition and are currently followed as outpatients. Unexpected sudden hemodynamic compromise could be due to coronary vasospasm, and this should be considered as one of the possible differential diagnoses. We were able to prevent the lethal consequences seen with coronary artery spasm by early diagnosis and management. (Int Heart J
\end{abstract} 2014; 55: 451-454)

Key words: Cardiac surgery, Coronary artery disease, Internal mammary artery, Extracorporeal membrane oxygenation

$\mathrm{C}$ oronary artery spasm (CAS) after off-pump coronary artery bypass surgery (CABG) is an uncommon complication, but can lead to serious consequences including death. Here, we report two patients who underwent off-pump CABG and experienced CAS that was confirmed by emergent coronary angiography and resulted in hemodynamic collapse in the immediate postoperative period. The possible etiologies, diagnosis, and treatments of CAS are discussed.

\section{Case Reports}

Surgery: In both cases, surgery was performed through a standard median sternotomy using bilateral internal mammary arteries (IMAs) harvested in a skeletonized fashion with an ultrasound scalpel (Harmonic Scalpel, Ethicon Endosurgery, Cincinnati, OH, USA). The IMAs were treated with intraluminal administration of papaverine to prevent vasospasm and were used as in situ grafts. Systemic heparin (300 IU/kg) was given, and the activated clotting time was maintained at over 300 seconds. The anastomosis sites were exposed and stabilized with the Starfish heart positioner (Medtronic, Minneapolis, MN, USA) and the Estech system (Estech, Danville, CA, USA). A silicone elastomer snare suture was placed proximal to the anastomosis site and tightened to occlude the targeted coronary artery before the arteriotomy. The suture was subsequently loosened after the placement of an intra-coronary shunt. A bloodless field was maintained with a carbon dioxide blower and the anastomoses were completed using continuous
8-0 Prolene sutures. Patency of the grafts was assessed using an ultrasonic flow meter which showed excellent flow in all grafts. After the anastomoses, heparin was fully reversed with protamine and the chest was closed with wires in a standard fashion.

Case 1: A 66 year-old male presented with a 2-month history of exertional angina and was admitted for an elective off-pump CABG. He had several risk factors for coronary disease including hypertension, tobacco abuse, and a family history of coronary disease. He was taking a nitrate, a beta-blocker, and a calcium channel blocker. Stress electrocardiogram (ECG) showed ST depression in the inferior leads (Figure 1A). Echocardiography showed preserved left ventricular systolic function with no significant valvular disease. Coronary angiography showed $90 \%$ stenosis of the left main coronary artery and $90 \%$ stenosis of the obtuse marginal artery (OM). The patient underwent a two-vessel off-pump CABG with the left internal mammary artery (LIMA) to the OM and the right internal mammary artery (RIMA) to the left anterior descending artery (LAD). Postoperatively, hemodynamics were stable on a dopamine infusion of $2.5 \mu \mathrm{g} / \mathrm{kg} /$ minute. Diltiazem and nitroglycerin drips were used to prevent CAS as per our routine.

Shortly after returning to the intensive care unit (ICU), the patient became acutely hypotensive, with systolic blood pressure of $74 \mathrm{mmHg}$. Central venous pressure dropped from 13 to $6 \mathrm{mmHg}$, and pulmonary artery diastolic pressure dropped from 19 to $12 \mathrm{mmHg}$. Hemodynamics improved after volume resuscitation and increased administration of inotropes $(10 \mu \mathrm{g} / \mathrm{kg} / \mathrm{minute}$ of dopamine and $10 \mu \mathrm{g} / \mathrm{kg} /$ minute of dob-

From the Department of ${ }^{1}$ Division of Cardiothoracic Surgery, Department of Surgery, Thomas Jefferson University, Philadelphia, USA, ${ }^{2}$ Department of Cardiovascular Surgery, Mitsui Memorial Hospital, Tokyo, ${ }^{3}$ Department of Cardiovascular Surgery, Totsuka Kyoritsu Second Hospital, and ${ }^{4}$ Department of Cardiovascular Surgery, St. Marianna University, Kanagawa, Japan.

Address for correspondence: Shinya Unai, MD, Division of Cardiothoracic Surgery, Department of Surgery, Thomas Jefferson University 1025 Walnut Street Room 607, Philadelphia, PA 19107, USA. E-mail: unai-tky@umin.ac.jp

Received for publication November 28, 2013. Revised and accepted February 4, 2014.

Released in advance online on J-STAGE July 28, 2014.

All rights reserved by the International Heart Journal Association. 


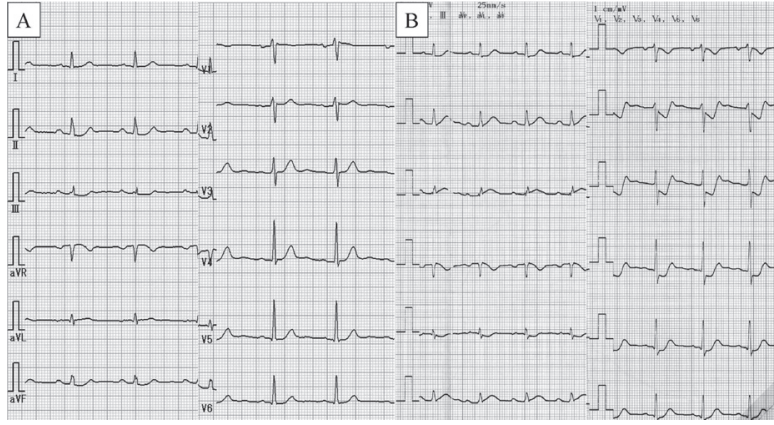

Figure 1. Preoperative (A) and postoperative (B) ECG from case 1.

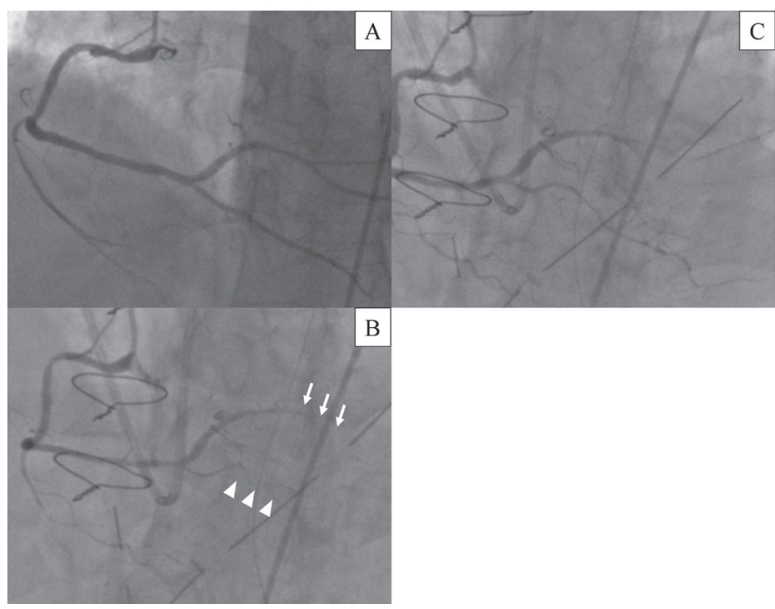

Figure 2. Preoperative angiography of the RCA showed no significant stenosis (A). Postoperative angiography showed complete occlusion of the posterior descending artery (arrowheads) and posterior left ventricular artery (arrows) (B). After intracoronary injection of isosorbide dinitrate and adenosine triphosphate $(\mathbf{C})$.

utamine), and cardiac index recovered to $3.0 \mathrm{~L} / \mathrm{s} / \mathrm{m}^{2}$. An ECG performed at this time showed new ST elevations in leads II, III, and aVF and ST depressions in V1-V6 (Figure 1B). The patient was immediately transported to the cardiac catheterization laboratory for angiography, which revealed patent grafts and $100 \%$ occlusion of the distal right coronary artery (Figure $2 \mathrm{~B}$ ), that was not seen on the preoperative catheterization. At this time the patient was diagnosed with CAS, and isosorbide dinitrate and adenosine triphosphate were administered directly to the coronary artery, resulting in resolution of the spasm (Figure 2C). Nicorandil drip was started in addition to diltiazem and nitroglycerin. Hemodynamics improved, and the patient was extubated on postoperative day 2 . The peak serum creatine kinase isoenzyme MB (CK-MB) was $147 \mathrm{U} / \mathrm{L}$ on postoperative day 1 . The patient was discharged home on postoperative day 31. Echocardiography before discharge showed good left ventricular function with no wall motion abnormalities.

Case 2: A 56-year-old male, with a history of hypertension, hyperlipidemia, type 2 diabetes, tobacco abuse, and a family history of coronary disease presented with chest tightness on exertion. His medications included an oral nitrate and a betablocker. Coronary angiography showed 50\% left main coronary artery disease and $90 \%$ stenosis of the OM (Figure 4A).

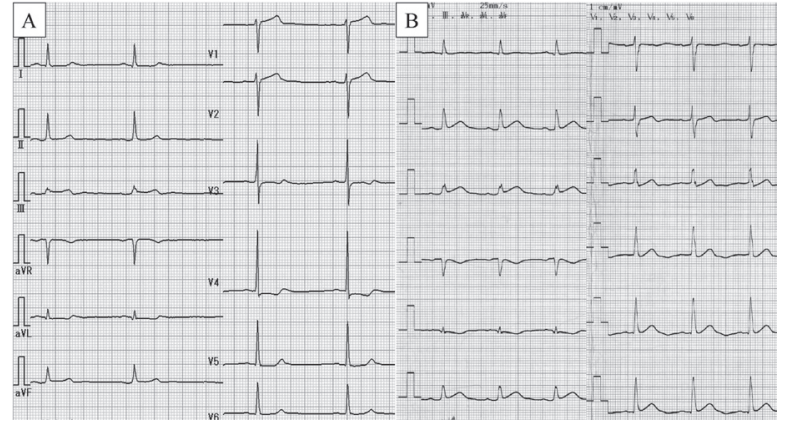

Figure 3. Preoperative (A) and postoperative (B) ECG from case 2.

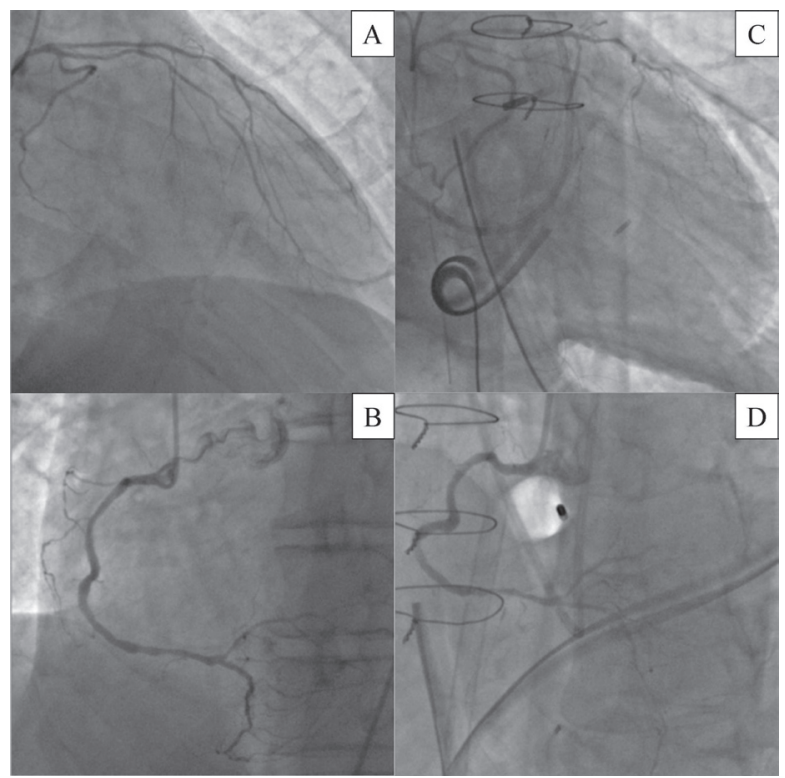

Figure 4. Preoperative angiography from case $2(\mathbf{A}, \mathbf{B})$. Postoperative angiography from case 2 shows diffuse spasm of the entire native coronary artery $(\mathbf{C}, \mathbf{D})$.

Echocardiography showed preserved left ventricular systolic function, and the patient was scheduled for an elective offpump CABG. He underwent a two-vessel off-pump CABG with the LIMA to the OM and RIMA to the LAD. He returned to the ICU in stable condition on dopamine ( $5 \mu \mathrm{g} / \mathrm{kg} /$ minute) and dobutamine $(2.5 \mu \mathrm{g} / \mathrm{kg} /$ minute $)$ in addition to diltiazem, nitroglycerin, and nicorandil infusion for prevention of spasm. An immediate postoperative ECG showed ST elevation in leads II, III and aVF (Figure 3B); however, hemodynamics were stable.

Approximately 3 hours after arrival at the ICU, upon waking from general anesthesia, the patient developed sustained ventricular fibrillation that did not respond to electrical defibrillation. An intra-aortic balloon pump (IABP) was emergently placed followed by extracorporeal membrane oxygenation (ECMO) for hemodynamic stability. Emergent coronary angiography showed patent grafts with diffuse vasospasm in the right and left coronary arteries (Figure 4C and D). Direct infusion of isosorbide dinitrate, nicorandil, and papaverine into the coronary arteries failed to improve the spasm. The patient returned to the ICU, and diltiazem, nicorandil, and nitroglycer- 
in drips were continued. Peak CK-MB was 224U/L on postoperative day 1. Left ventricular function gradually recovered, and ECMO and IABP were discontinued on postoperative day 4 and 6 , respectively. The patient was extubated on postoperative day 8 and discharged home on postoperative day 84, after an extensive period of in-hospital cardiac rehabilitation. Echocardiography performed before discharge showed mildly decreased left ventricular systolic function with anterior/lateral wall hypokinesis.

\section{Discussion}

The reported incidence of CAS after cardiac surgery ranges from 0.8 to $1.3 \%$; however, this may be an underestimation because the diagnosis requires coronary angiography. ${ }^{1)}$ Once CAS occurs, the risk of postoperative mortality is as high as 10 to $50 \%$. ${ }^{1)}$ From January 2001 to March 2011, a total of 644 CABG were performed at Mitsui Memorial Hospital. Among them, 289 cases (44.9\%) were off-pump CABG and there were two cases of postoperative CAS, giving an incidence rate of $0.69 \%$.

Etiology: The possible causes of postoperative CAS have been discussed in the literature. Patients who have preoperative findings or symptoms of Prinzmetal's variant angina may have a predilection to post-CABG CAS; however, most case reports have not mentioned a history of CAS. ${ }^{2)}$ Smoking is a major risk factor for CAS in the absence of significant coronary stenosis. ${ }^{3)}$ Intraoperative causes of CAS include manipulation of the heart by a topical suction device and deep pericardial suture during an anastomosis, ${ }^{4}$ intimal injury caused by a carbon dioxide blower ${ }^{5)}$ and intracoronary shunts, ${ }^{6,7)}$ external traction and compression on the arterial wall caused by an elastic snare, ${ }^{8,9)}$ compression caused by chest tubes, ${ }^{10)}$ and trauma to the coronary arteries during cannulation. ${ }^{4)}$ Electrolyte abnormalities such as hypomagnesaemia ${ }^{11,12)}$ and hyperkalemia ${ }^{13)}$ may also contribute to CAS. Hypomagnesaemia impairs the release of nitric oxide, a vasodilator and inhibitor of platelet aggregation, from the coronary endothelium. ${ }^{12)}$ In on-pump CABG, magnesium is included in the cardioplegia solution and may have a protective effect against CAS. In off-pump $\mathrm{CABG}$, the serum magnesium level may be lowered by the rapid infusion of fluid without magnesium supplementation. ${ }^{14)}$ There have been reports of lethal spasm after both on- and offpump CABG, and the magnesium levels were low even in the on-pump cases. ${ }^{1)}$ Release of vasoconstrictive agents, such as serotonin and thromboxane released by damaged platelets ${ }^{15}$ and histamine released owing to allergic reactions to protamine and blood products, ${ }^{16)}$ may also trigger CAS. Infusion of cardioplegia at a high pressure may make the coronary arteries susceptible to vasospastic stimuli. ${ }^{17)}$ Lamping, et al reported that elevation of coronary artery perfusion pressure for more than one minute resulted in an increased vasoconstrictor response to serotonin that persisted for at least 2.5 hours. ${ }^{18)}$ High endogenous catecholamine levels during waking from anesthesia and hypoxic events ${ }^{1920)}$ and high exogenous catecholamine levels, particularly dopamine, have been shown to precipitate CAS in susceptible patients. ${ }^{21)}$ Respiratory alkalosis due to hyperventilation, ${ }^{17,22)}$ hypothermia, ${ }^{23)}$ and underlying atherosclerotic disease, ${ }^{24)}$ are also reported to contribute to CAS.

In both of our cases, history of tobacco abuse may have resulted in a predilection towards CAS. Intraoperative manipulation of the heart and intimal injury during the procedure, hypomagnesaemia (although this was not measured postoperatively), catecholamine surge, and a sympathetic nervous system response while waking in the ICU, may have also contributed to the occurrence of CAS in our patients.

Diagnosis: If not promptly diagnosed and treated, CAS may result in myocardial infarction with life-threatening consequences. Clinically, post-CABG CAS may become apparent through a change in the ECG, hemodynamic instability, arrhythmia, circulatory collapse, or cardiac arrest. Echocardiography may show new segmental wall motion abnormalities that were not seen at the time of the pre-operative study. However, the exact diagnosis of CAS can be reached only by coronary angiography.

CAS may occur intraoperatively, ${ }^{25)}$ immediately after returning to the ICU, ${ }^{26)}$ upon awakening, ${ }^{8,20)}$ or shortly after extubation. ${ }^{14)}$ In previous studies, the majority of CAS cases have occurred within the first 24 hours after the operation. ${ }^{27,28)}$ Clinicians must maintain a high index of suspicion for CAS when a patient develops an unexplainable postoperative hemodynamic instability. In such situations, after excluding other possible causes of hemodynamic instability such as cardiac tamponade and tension pneumothorax, coronary angiography should be considered. The RCA is most commonly involved in CAS; ${ }^{1)}$ however, other native coronary arteries as well as the IMA graft or the saphenous vein graft used for bypass may also be involved. $^{27,29)}$ Surgical re-exploration has no role in CAS, and may lead to fatal consequences owing to prolonging the critical time for catheterization treatment.

Treatment: The best treatment for CAS is intravenous injection of vasodilator agents such as isosorbide dinitrate, adenosine triphosphate, papaverine, nicorandil, and calcium channel blockers. However, CAS may cause extremely strong contraction of the vessels, and consequently very high concentrations of vasodilators are required to relieve the spasm. ${ }^{9)}$ Therefore intracoronary injection of vasodilators during coronary angiography is the most effective treatment for CAS, and indeed could be lifesaving. ${ }^{25)}$

Several studies have reported that stent implantation is an option in cases of refractory CAS with a focal lesion; ${ }^{30,31)}$ however, stenting in patients with CAS is associated with complications such as stent-edge spasm and stent crush phenomenon and therefore should be carefully considered. ${ }^{32,33)}$ If CAS is intraoperatively identified, it may be treated by drug infusion directly through the bypass graft. ${ }^{25}$ )

In hemodynamically unstable patients, the use of an IABP can increase the coronary flow and support the struggling left ventricle. In cases of severe cardiogenic shock or cardiac arrest, however, an IABP will not be sufficient to support the patient's hemodynamics, and more advanced mechanical circulatory support such as veno-arterial ECMO should be performed. After initiation of ECMO, catecholamine infusions which may be aggravating the CAS by the alpha-adrenergic effect can be discontinued, since ECMO can provide full circulatory support. In addition, ECMO will improve oxygenation, decompress the heart and reduce myocardial oxygen consumption, therefore reducing any on-going ischemia. Once stabilized on ECMO, the patient can be safely transported to the catheterization laboratory for further evaluation. Support with ECMO combined with continuous use of intravenous va- 
sodilators for spasm could be lifesaving in severe CAS cases, as shown in case 2 .

Conclusion: We presented two cases of hemodynamically significant post-CABG CAS that were successfully treated in different ways. Although a definite diagnosis of CAS is difficult to establish, CAS should always be considered as a possible cause of hemodynamic compromise after CABG. Preoperative recognition of high-risk patients, avoiding excessive manipulation of the heart, minimizing the use of a carbon dioxide blower, applying a minimal amount of traction to the elastic snare, and avoiding hypothermia are all essential for the prevention of CAS. The routine use of inotropic agents, especially dopamine, is not recommended in patients who are at high risk of developing CAS. Postoperatively, intravenous nitroglycerin, calcium antagonists, infusion of supplemental magnesium, the use of adequate sedation, and minimizing the amount of stimulation while wakening the patient may decrease the risk of the CAS. Once CAS is suspected, immediate coronary angiography is essential for diagnosis and treatment.

\section{REFERENCES}

1. Buxton AE, Goldberg S, Harken A, Hirshfield J Jr, Kastor JA Coronary-artery spasm immediately following coronary after myocardial revascularization: recognition and management. $\mathrm{N}$ Engl J Med 1981; 304: 1249-53.

2. Buxton AE, Hirshfeld JW Jr, Untereker WJ, et al. Perioperative coronary arterial spasm: long-term follow-up. Am J Cardiol 1982; 50: 444-51.

3. Yasue H, Nakagawa H, Itoh T, Harada E, Mizuno Y. Coronary artery spasm--clinical features, diagnosis, pathogenesis, and treatment. J Cardiol 2008; 51: 2-17. (Review)

4. Zeff RH, Iannone LA, Kongtahworn C, et al. Coronary artery spasm following coronary artery revascularization. Ann Thorac Surg 1982; 34: 196-200.

5. Okazaki Y, Takarabe K, Murayama J, et al. Coronary endothelial damage during off-pump CABG related to coronary-clamping and gas insufflation. Eur J Cardiothorac Surg 2001; 19: 834-9.

6. Hangler HB, Pfaller K, Ruttmann E, et al. Effects of intracoronary shunts on coronary endothelial coating in the human beating heart. Ann Thorac Surg 2004; 77: 776-80.

7. Izutani $\mathrm{H}$ and Gill IS. Acute graft failure caused by an intracoronary shunt in minimally invasive direct coronary artery bypass grafting. J Thorac Cardiovasc Surg 2003; 125: 723-4.

8. Trimboli S, Oppido G, Santini F, Mazzucco A. Coronary artery spasm after off-pump coronary artery by-pass grafting. Eur J Cardiothorac Surg 2003; 24: 830-3.

9. Lin CY, Weng ZC, Loh SH, Hong GJ, Tsai CS. Coronary artery spasm after off-pump coronary artery bypass grafting. ANZ J Surg 2007; 77: 126-9.

10. Marques KM, Jansen EE, Huybregts RA. Images in cardiology. Profound coronary spasm caused by a mediastinal draining tube. Heart 2006; 92: 514.

11. Sueda S, Fukuda H, Watanabe K, et al. Magnesium deficiency in patients with recent myocardial infarction and provoked coronary artery spasm. Jpn Circ J 2001; 65: 643-8.

12. Pearson PJ, Evora PR, Seccombe JF, Schaff HV. Hypomagnesemia inhibits nitric oxide release from coronary endothelium: protective role of magnesium infusion after cardiac operations. Ann Thorac Surg 1998; 65: 967-72.
13. Perez JE, Saffitz JE, Gutierrez FA, Henry PD. Coronary artery spasm in intact dogs induced by potassium and serotonin. Circ Res 1983; 52: 423-31.

14. Ueno T, Ikeda K, Nakashima A. Vasodilator resistant lethal spasm after uncomplicated off-pump coronary surgery. Asian Cardiovasc Thorac Ann 2006; 14: 158-60.

15. Ellis EF, Oelz O, Roberts LJ 2nd, et al. Coronary arterial smooth muscle contraction by a substance released from platelets: evidence that it is thromboxane A2. Science 1976; 193: 1135-7.

16. Ginsburg R, Bristow MR, Kantrowitz N, Baim DS, Harrison DC. Histamine provocation of clinical coronary artery spasm: implications concerning pathogenesis of variant angina pectoris. Am Heart J 1981; 102: 819-22.

17. Lemmer JH Jr, Kirsh MM. Coronary artery spasm following coronary artery surgery. Ann Thorac Surg 1988; 46: 108-15. (Review)

18. Lamping KG, Dole WP. Acute hypertension selectively potentiates constrictor responses of large coronary arteries to serotonin by altering endothelial function in vivo. Circ Res 1987; 61: 904-13.

19. Bittner HB. Coronary artery spasm and ventricular fibrillation after off-pump coronary surgery. Ann Thorac Surg 2002; 73: 297 300 .

20. Singla MK, Mukherjee KC, Shrivastava A, Goyal V. Successful management of potentially fatal vasodilator-resistant spasm of a nongrafted coronary artery. J Cardiothorac Vasc Anesth 2011; 25: 1104-5.

21. Crea F, Chierchia S, Kaski JC, et al. Provocation of coronary spasm by dopamine in patients with active variant angina pectoris. Circulation 1986; 74: 262-9.

22. Yasue H, Nagao M, Omote S, Takizawa A, Miwa K, Tanaka S. Coronary arterial spasm and Prinzmetal's variant form of angina induced by hyperventilation and Tris-buffer infusion. Circulation 1978; 58: 56-62.

23. Yamashina Y, Yagi T, Namekawa A, et al. Ventricular fibrillation caused by coronary artery spasm during mild therapeutic hypothermia after resuscitation. Am J Emerg Med 2013; 31: 453.

24. Lanza GA, Careri G, Crea F. Mechanisms of coronary artery spasm. Circulation 2011; 124: 1774-82. (Review)

25. Mayumi H, Kawachi Y, Kohno H, et al. Drug infusion through a branch of the aortocoronary vein graft for refractory coronary spasm. Ann Thorac Surg 1994; 58: 869-71.

26. Bossert T, Bittner HB, Gummert JF, Mohr FW. Coronary artery spasm of the native right coronary artery during off-pump coronary surgery of the left coronary artery system. Clin Res Cardiol 2006; 95: 115-8.

27. He GW, Fan KY, Chiu SW, Chow WH. Injection of vasodilators into arterial grafts through cardiac catheter to relieve spasm. Ann Thorac Surg 2000; 69: 625-8.

28. Hosoba S, Suzuki T, Takashima N, et al. Successful management of refractory lethal coronary spasm after off-pump coronary bypass grafting. Ann Thorac Cardiovasc Surg 2012; 18: 359-62.

29. D’Souza VJ, Velasquez G, Kahl FR, Hackshaw BT, Amplatz K. Spasm of the aortocoronary venous graft. Radiology 1984; 151: 83-4.

30. Khatri S, Webb JG, Carere RG, Dodek A. Stenting for coronary artery spasm. Catheter Cardiovasc Interv 2002; 56: 16-20.

31. Schena S, Wildes T, Beardslee MA, Lasala JM, Damiano RJ Jr, Lawton JS. Successful management of unremitting spasm of the nongrafted right coronary artery after off-pump coronary artery bypass grafting. J Thorac Cardiovasc Surg 2007; 133: 1649-50.

32. Kaku B, Kanaya H, Horita Y. Spontaneous stent-edge spasm in a patient with myocardial infarction. Heart 2005; 91: 937.

33. Yoshida T, Kobayashi Y, Nakayama T, Kuroda N, Komiyama N, Komuro I. Stent deformity caused by coronary artery spasm. Circ J 2006; 70: 800-1. 